\title{
Assessment of Examination Stress on Working Memory in Medical Students
}

\author{
Sunil Kumar Jena ${ }^{1}$, Manoranjan Acharya ${ }^{2, *}$
}

\section{Sunil Kumar Jena', Manoranjan Acharya ${ }^{2, *}$}

'Assistant Professor, Department of Physiology, VIMSAR, Burla, Odisha, INDIA.

${ }^{2}$ Professor \& HOD, Department of Neurology, VIMSAR, Burla, Odisha, INDIA.

\section{*Correspondence}

Prof. Dr. Manoranjan Acharya Professor \& HOD, Department of Neurology, Veer Surendra Sai Institute of Medical Sciences And Research (VIMSAR), Burla-768017, Odisha, INDIA.

Phone: +919437170200

Email: manoranjanneuro@gmail.com History

- Submission Date: 29-12-2019;

- Review completed: 14-02-2020;

- Accepted Date: 28-02-2020

DOI : 10.5530/ijcep.2020.7.1.4

Article Available online

http://www.ijcep.org/

Copyright

(C) 2020 Phcog.Net. This is an openaccess article distributed under the terms of the Creative Commons Attribution 4.0 International license.

\begin{abstract}
Background and Aim: Working memory has substantial importance in academic, professional and social aspects of life. Working memory deficit influences the academic performance of students irrespective of their intelligence quotient (IQ) status. Therefore, this study was designed to evaluate the working memory status owing to examination stress. Methods: Forty healthy subjects were recruited for this study and it was conducted after ethical approval from institutional ethical committee. Written consent was obtained from all participants. EEG was done in 67 students and 40 students shown $\alpha$ wave on EEG were selected as study subjects. Digit Span Forward (DSF), Digit Span Backward (DSB) and EEG was determined both in relax and in examination stress. Digit span and EEG was compared in relaxed and stress state. Results: Mean frequencies of EEG in relax was 9.6 Hz with SD 0.86 while in stress it was $25.9 \mathrm{~Hz}$ with SD 0.31. DSF in stress was $6.45 \pm 1.15$ and in relax state it was $7.42 \pm 1.21$. Mean DSB in stress was $5.7 \pm 0.64$ and in relaxed period it was 7.1 \pm 0.74 . In stress there was negative correlation between digit span and EEG. Conclusion: This study concluded that examination stress was associated with low DSF and DSB. Negative correlation between digit span and EEG suggested examination stress was a factor of working memory deficit.

Key words: Forward digit span, Backward digit span, Electroencephalogram, Examination stress, Working memory.
\end{abstract}

\section{INTRODUCTION}

Now-a-days a lot of interest has been shown by researchers in working memory and enlightens the importance of working memory in academic, professional and social life. ${ }^{[1]}$ Study of working memory capacity has substantial relationship with performance in cognitive tasks. ${ }^{[2]}$ Working memory is the ability of a person to store and recall very short-term information, words, numbers, figures etc. ${ }^{[3]}$ Working memory measures the capacity of a person to acquire knowledge rather than measuring what the person has already learned. ${ }^{[4]}$ Study has done first on animal about the impact of acute and chronic stress on working memory and also has proved that stress can impair the working memory. ${ }^{[5]}$ Prefrontal cortex (PFC) is the site of brain where catecholamine is released in stress. This catecholamine is responsible to impair working memory performance by rapidly decreasing the neuronal firing in PFC through feed forward and intracellular signaling pathways. ${ }^{[6]} \mathrm{Ex}$ posure to chronic stress changes the architecture of PFC, including the dendritic atrophy and spine loss leading to profound working memory deficits. ${ }^{[7]} \mathrm{Low}$ PFC functional connectivity is found in medical students undergoing stressful examination. ${ }^{[8]}$ Beta wave in EEG shows significant relation with high stress while alpha wave shows significant relation with relaxed state of brain. ${ }^{[9]}$ Medical students face to stress situations like academic, social, cultural etc. and one of the most consistent event is examination stress. Therefore, this study was conceptualized to observe the working memory owing to examination stress. The objective of this study was to evaluate the effect of examination stress on working memory in medical students.

\section{MATERIALS AND METHODS}

This descriptive cross sectional study was designed and conducted in department of Physiology in collaboration with Neurology in Veer Surendra Sai Institute of Medical Sciences and Research (VIMSAR), Burla, Odisha. It was accomplished between Jan to June 2019. This study protocol was approved by Institutional Ethical Committee of VIMSAR. Forty male MBBS students were recruited as the subjects for this study. All subjects were between the age ranges of 18 to 20 years. Apparently healthy students were selected for this study. Students suffering from any chronic disease or history of taking drugs were excluded from the study. When there was not any examination students were screened for selection of subjects. Written consent was taken and 67 male students were screened for electroencephalogram (EEG) after their taking written consent. Only male 
subjects were selected to avoid the influence of female hormones that fluctuate during menstrual cycle which in turn might have an effect on EEG. They were well explained about the purpose and output of study. Among 67 students we selected 40 students having alpha wave (frequency 8-13) in EEG. This screening procedure confirmed that selected 40 students were in relaxed state when there was no examination. ${ }^{[9]}$ Simultaneously their working memory was assessed in relaxed period. All 40 subjects were again assessed for working memory during the period of examination and their EEG was recorded.

\section{Recording of EEG}

Electrodes used for EEG recording were silver-silver chloride discs of 4-10 mm in diameter. ${ }^{[10]}$ Electrodes were positioned according to international 10-20 system to record EEG. This system included total 21 electrodes out of which 19 put on scalp and 2 on both sides ear lobes. ${ }^{[1]}$ EEG recording was done in eye closed condition for $10 \mathrm{~min}$ to observe the different wave pattern as per the protocol laid down by International Federation of Clinical Neurophysiology. ${ }^{[12]}$ Eye close EEG was chosen to minimize the effect of sensory input and effect of movement on brain activity. ${ }^{[13]}$

\section{Digit Span}

Digit span, a subset of Wechsler Adult Intelligence Scale-Fourth Edition (WAIS-IV) was administered to assess the working memory capacity. ${ }^{[14]}$ Two subsets of digit span i.e. digit span forward (DSF) and digit span backward (DSB) were administered for working memory assessment. In DSF examiner read a sequence of numbers and examinee recalled the numbers in same order. For example when examiner read 35764, the examinee recalled 35764. In DSB when examiner read 35764, the examinee recalled 46753. For each subject two trials were done and the highest digits counted was selected for recorded according to (WAIS-IV) manual. ${ }^{[14]}$ DSF and DSB was assessed in both relax and examination stress.

\section{Statistical Analysis of Data}

Statistical analysis was done by statistical software SPSS 20 IBM Corporation. Statistical test used were Paired $t$ test and Pearson correlation. $P$ value $<0.05$ was considered to be significant. Digit span and EEG change in relaxed and stress state was analyzed by paired t test. Pearson correlation was used to observe the association between EEG and digit span.

\section{RESULTS}

This study was accomplished among 40 healthy subjects to evaluate the effect of examination stress on working memory. Table 1 depicts the change in EEG in relax and stress state. In relax state frequency of EEG was $9.6 \mathrm{~Hz}$ with SD 0.86 while in stress it was 25.9 with SD 0.31. Data was analyzed by paired $t$ test and change was found significant $(P=0.000)$. Table 2 depicts the DSF and DSB of subjects in relax and stress state. Mean DSF in relax state was 7.42 with SD 1.21 while in stress it was 6.45 with SD 1.15. Data comparison was done by paired $t$ test and difference was found significant $(P=0.001)$. Mean DSB in relaxed state was 7.1 with SD 0.74 while in stress it was 5.7 with SD 0.64 . Data comparison was done by paired $t$ test and difference was found significant $(\mathrm{P}=0.000)$. Table 3 and Figure 1, Figure 2, depicts the correlation between digit span and EEG. In stress there was negative correlation between DSF and EEG frequency, Pearson correlation coefficient " $\mathrm{r}$ " was $-0.963(\mathrm{P}=0.000)$. In stress there was negative correlation between DSB and EEG frequency, Pearson correlation coefficient " $\mathrm{r}$ " was $-0.490(\mathrm{P}=0.001)$.

\section{DISCUSSION}

School and college students are exposed to stressors like academic, social, cultural etc. Examination is an important academic stressor in a student's life. Vast course of medical curriculum put an extra load on student's life. Therefore, it is difficult to achieve a good score in examination and to achieve their target. ${ }^{[15]}$ So examination act as a substantial stressor on medical student's life. During the period of examination, most of medical students perceive a lot of stress due to course burden. ${ }^{[15]}$

EEG wave analysis in this study provided information about the functional status of the brain. Baseline EEG was done and students showing alpha wave $(8-13 \mathrm{~Hz})$ on EEG were selected as the study subjects. When subjects were not exposed to examination their EEG wave was alpha and they were in relaxed state. ${ }^{[9]}$ In this study we found that in examination stress the EEG wave was high frequency $(13-30 \mathrm{~Hz})$ beta pattern type. Previous studies suggested that beta wave in EEG indicates that the person is in stress whereas alpha wave indicates relaxed state. ${ }^{[9,16]}$ Thus analysis of EEG confirmed that examination put stress on subjects, which was reflected by EEG frequency that shown the functional status of brain.

Richard SL et al. in their research on examination stress put their view that DSB was increased in stress while DSF was not affected. ${ }^{[17]}$ Our study contradicted to their study i.e. both DSF and DSB were decreased owing to examination stress. Study of Klein and Boals suggested that working memory capacity was decreased owing to stress. ${ }^{[18]}$ Their study correlated to our study. Vedhara et al. and Kuhlman et al. suggested that stress was not a factor that affect the working memory. ${ }^{[19,20]}$ Their was contradicted to our study. Researchers Elzinga and Roelofsin in their study suggested that the DSF was low owing to stress. ${ }^{[21]}$ Their study was correlated to our study.

Correlation analysis found that there was negative correlation between digit span and EEG in examination stress. This suggested that at higher brain activity i.e. high frequency EEG, digit span was low. Researchers suggested that high frequency EEG may be due to maintenance of verbal stimuli in auditory memory. Working memory is associated with mental processing and manipulation of information. For manipulation vol-

\section{Table 1: Comparison of EEG.}

\begin{tabular}{cccc} 
Variables & Relax & Stress & $P$ \\
& Mean \pm SD & Mean \pm SD & \\
\hline EEG frequency & $9.6 \pm 0.86$ & $25.9 \pm 0.31$ & 0.000
\end{tabular}

Data comparison by paired $t$-test, $\mathrm{P}$ value significant at $<0.05$,

EEG-Electroencephalogram, SD-Standard deviation

Table 2: Comparison of Digit Span.

$\begin{array}{cccc}\text { Variables } & \begin{array}{c}\text { Relax } \\ \text { Mean } \pm \text { SD }\end{array} & \begin{array}{c}\text { Stress } \\ \text { Mean } \pm \text { SD }\end{array} & P \\ \text { DSF } & 7.42 \pm 1.21 & 6.45 \pm 1.15 & 0.001 \\ \text { DSB } & 7.1 \pm 0.74 & 5.7 \pm 0.64 & 0.000\end{array}$

Data comparison by paired $t$ test, $P$ value significant at $<0.05$, DSF- Digit Span Forward, DSB- Digit Span Backward, SD-Standard deviation

Table 3: Correlation between Digit Span and EEG.

\begin{tabular}{|cccc|}
\hline \multicolumn{1}{c}{ Variables } & Correlation Coefficient $(r)$ & $P$ \\
\hline \multirow{2}{*}{ Stress } & DSF vs. EEG & -0.963 & 0.000 \\
& DSB vs. EEG & -0.490 & 0.001
\end{tabular}

Data analysis by Pearson correlation, $P$ value significant at $<0.01$, DSF- Digit Span Forward, DSB- Digit Span Backward,

EEG-Electroencephalogram 


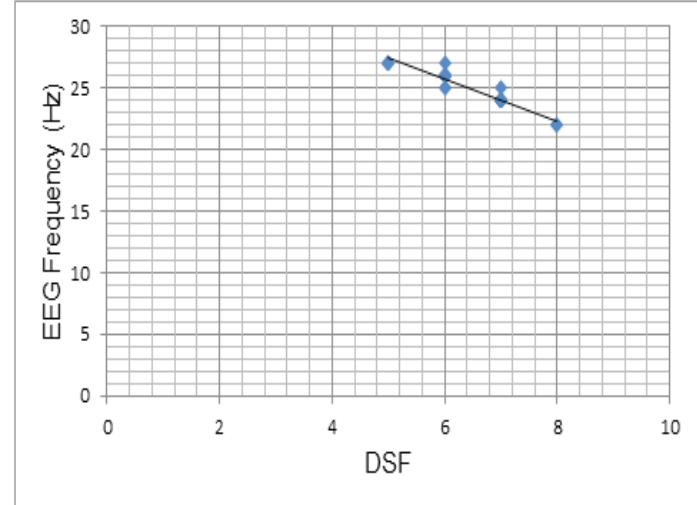

Figure 1: Correlation between EEG and DSF.

EEG:Electroencephalogram, DSF:Digit Span Forward

ume of information is so high that may be the cause of high frequency beta wave EEG. ${ }^{[22,23]}$ In examination stress students are more attentive for which their alertness is increased as well as they are anxiety level is increased. Therefore, their EEG pattern is shifted to beta pattern. ${ }^{[24]}$

Stress induced release of catecholamine from prefrontal cortex (PFC) rapidly decreases its neuronal firing and impairs working memory performance through feed forward, intracellular signaling pathways. ${ }^{[6]}$ There is a link between working memory capacity and neural ability to focus attention on task relevant information and to ignore distraction. ${ }^{[25]}$ Working memory deficit has severe detrimental effect on academic performance of students. Researchers suggested that one in every ten students have low or deficit working memory. Among them majority have poor academic performance independent of their IQ status. ${ }^{[26]}$ There is a link between cognitive aspect of brain and working memory. Therefore, working memory impairment or deficit is associated with low academic performance and achievements for students. ${ }^{[27]}$

\section{Limitations of the Study}

Hormonal assessment is an important indicator of stress was not estimated. Only male subjects were recruited and effect of examination stress on working memory in female could not be evaluated. Only frequency of EEG was taken into consideration for convenience.

\section{CONCLUSION}

This study concluded that DSF and DSB both decreased owing to examination stress in comparison to relax state. There was negative correlation between digit span (both DSF and DSB) and EEG. Thus, in examination stress there was working memory deficit.

\section{ACKNOWLEDGEMENT}

It is our immense pleasure to acknowledge the subjects participated in this valuable study, without whom it was not completed. We are also thankful to technicians of Neurology department for their cooperation towards this work.

\section{CONFLICT OF INTEREST}

The authors declare they have no conflict of interest.

\section{ABBREVIATIONS}

IQ: Intelligent Quotient; DSF: Digit Span Forward; DSB: Digit Span Backward; PFC: Prefrontal cortex; EEG: Electroencephalogram; WAISIV: Wechsler Adult Intelligence Scale-Fourth Edition.

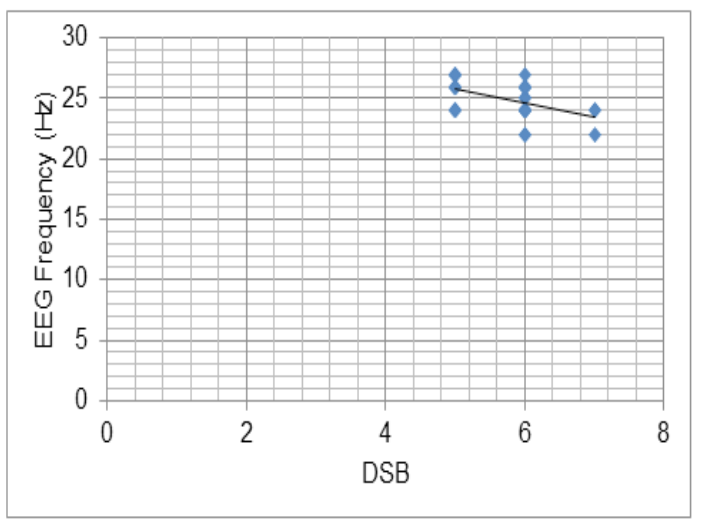

Figure 2: Correlation between EEG and DSB.

EEG:Electroencephalogram, DSB:Digit Span Backward

\section{REFERENCES}

1. Cogmed Working Memory Training. 2011. Available from: http://www.cogmed. $\mathrm{com} /$

2. Conway AR, Kane MJ, Engle RW. Working memory capacity and its relation to general intelligence. Trends Cogn Sci. 2003;7(12):547-52.

3. Pisoni DB, Cleary M. Measures of working memory span and verbal rehearsal speed in deaf children after cochlear implantation. Ear Hear. 2003;24(1 Suppl):106S-20S.

4. Alloway TP, Passolunghi MC. The relations between working memory and ar ithmetical abilities: A comparison between Italian and British children. Learn Individ Differ. 2011;21(1):133-7.

5. Arnsten AF. The biology of being frazzled. Science. 1998;280(5370):1711-2.

6. Arnsten AF. Stress signalling pathways that impair prefrontal cortex structure and function. Nat Rev Neurosci. 2009;10(6):410-22.

7. Radley JJ, Rocher AB, Miller M, Janssen WG, Liston C, Hof PR, et al. Repeated stress induces dendritic spine loss in the rat medial prefrontal cortex. Cereb Cortex. 2006;16(3):313-20.

8. Liston C, McEwen BS, Casey BJ. Psychosocial stress reversibly disrupts prefrontal processing and attentional control. Proc Natl Acad Sci U S A. 2009;106(3):912-7.

9. Gupta RK, Rajeswaran J, Bhattacharya A, Ojha A, Bennett CN. Academic Stress in School Children: Behavioural and Electrophysiological Outcomes Study. Indian J Clin Psychol. 2018;45(2):18-22.

10. Barlow JS, Kamp A, Morton HB, Ripoche A, Shipton H, Tchavdarov DB. EEG instrumentation standards: Report of the committee on EEG instrumentation standards of the international federation of societies for electroencephalography and clinical neurophysiology. Electroencephalogra Clin Neurophysiol. 1978:44:144-50

11. Guideline thirteen: Guidelines for standard electrode position nomenclature. American Electroencephalographic Society. J Clin Neurophysiol. 1994;11(1):111 3.

12. Recommendations for the practice of clinical neurophysiology: Guidelines of the International Federation of Clinical Neurophysiology. Electroencephalogr Clin Neurophysiol Suppl. 1999;52:1-304

13. Kondakor I, Brandeis D, Wackermann J, Kochi K, Koenig T, Frei E, et al. Multichannel EEG fields during and without visual input: Frequency domain model source locations and dimensional complexities. Neurosci Lett. 1997;226(1):49 52.

14. David EH. Wechsler Adult Intelligence Scale IV (WAIS IV): Return of the Gold Standard. Appl Neuropsychol. 2009;16(1):85-7.

15. Jena SK, Misra AK, Mohanty A. Effect of examination stress on blood sugar in medical students. CHRISMED J Health Res. 2016;3(4):268-72.

16. Jena SK. Examination stress and its effect on EEG. Int J Med Sci Public Health. 2015;11(4):1493-7.

17. Richard SL, Ani N, Dennis JC, Nicole YW. Examination stress and components of working memory. Stress. 2008;11(4):108-14.

18. Klein K, Boals A. The relationship of life event stress and working memory capacity. Appl Cogn Psychol. 2001;15(5):565-79.

19. Kuhlmann S, Piel M, Wolf OT. Impaired memory retrieval after psychosocial stress in healthy young men. J Neurosci. 2005;25(11):2977-82.

20. Vedhara K, Hyde J, Gilchrist ID, Tytherleigh M, Plummer S. Acute stress, memory, attention and cortisol. Psychoneuroendocrinology. 2000;25(6):535-49.

21. Elzinga BM, Roelofs K. Cortisol-induced impairments of working memory require acute sympathetic activation. Behav Neurosci. 2005;119(1):98-103. 
22. Spitzer B, Blankenburg F. Supramodal parametric working memory processing in humans. J Neurosci. 2012;32(10):3287-95.

23. Spitzer B, Gloel M, Schmidt TT, Blankenburg F. Working memory coding of analog stimulus properties in the human prefrontal cortex. Cereb Cortex. 2014;24(8):2229-36

24. Papanicolaou AC, Loring DW, Deutsch G, Eisenberg HM. Task-related EEG asymmetries: A comparison of alpha blocking and beta enhancement. Int $J$ Neurosci. 1986;30(1-2):81-5
25. Zanto TP, Gazzaley A. Neural suppression of irrelevant information underlies optimal working memory performance. J Neurosci. 2009;29(10):3059-66.

26. Alloway TP, Gathercole SE, Kirkwood H, Elliott J. The cognitive and behavioral characteristics of children with low working memory. Child Dev. 2009;80(2):60621.

27. Alloway TP. Working Memory, but Not IQ, Predicts Subsequent Learning in Children with Learning Difficulties. Eur J Psychol Assess. 2009;25(2):92-8.

Cite this article: Jena SK, Acharya M. Assessment of Examination Stress on Working Memory in Medical Students. Int J Clin Exp Physiol. 2020;7(1):14-7. 